\title{
Evaluation of Stereo Matching Costs on Close Range, Aerial and Satellite Images
}

\author{
Ke Zhu ${ }^{1}$, Pablo d'Angelo ${ }^{2}$ and Matthias Butenuth ${ }^{1}$ \\ ${ }^{1}$ Remote Sensing Technology, Technische Universität München, Arcisstr 21, München, Germany \\ ${ }^{2}$ The Remote Sensing Technology Institute, German Aerospace Center, Oberpfaffenhofen, Germany \\ \{ke.zhu,matthias.butenuth\}@bv.tum.de,pablo.angelo@dlr.de
}

\begin{abstract}
Keywords: dense stereo matching, cost function, performance, observation constrain
Abstract: In the last years, most dense stereo matching methods use evaluation on the Middlebury stereo vision benchmark datasets. Most recent stereo algorithms were designed to perform well on these close range stereo datasets with relatively small baselines and good radiometric behaviour. In this paper, different matching costs on the Semi-Global Matching algorithm are evaluated and compared using the common Middlebury datasets, aerial and satellite datasets with ground truth. The experimental results show that the performance of dense stereo methods for datasets with larger baselines and stronger radiometric changes relies on even more robust matching costs. In addition, a novel matching cost based on mutual information and Census is introduced showing the most robust performance on close range, aerial and satellite data.
\end{abstract}

\section{INTRODUCTION}

The performance of dense stereo matching methods depends on all components, this includes preprocessing, matching costs, aggregation, disparity optimization and postprocessing steps. Most work on dense stereo uses well known cost functions such as absolute differences or Birchfied-Tomasi (Birchfield and Tomasi, 1998), as these perform well on the Middlebury datasets (Scharstein and Szeliski, 2002; Scharstein and Szeliski, 2011). The most intuitive cost assumes the consistency between intensities of two corresponding pixels. Using different matching costs, like Absolute Differences (AD), Mutual Information (MI) (Viola and Wells, 1997; Chrastek and Jan, 1997) or Census (Zabih and Woodfill, 1994) on the same stereo matching method can generate very different results (Hirschmüller and Scharstein, 2009; Neilso and Yang, 2008).

Dense stereo algorithms are typically evaluated with a small baseline configuration, artificial and often ambient light sources. Radiometric changes due to vignetting, gamma changes etc. were often simulated by modifying these small baseline images (Hirschmüller and Scharstein, 2009; Neilso and Yang, 2008). These simulations do not capture all effects such as non-lambertian reflectance. In the evaluation of stereo matching costs using the Middlebury data (Hirschmüller and Scharstein, 2009): Cen- sus shows the best and the most robust overall performance. Mutual information performs very well with global methods. On radiometrically distorted Middlebury datasets, and datasets with varying illumination, Census and Mutual Information outperform AD clearly. But we are not aware of matching cost performance evaluation for images with larger baselines and remotely sensed images.

In this study, the Semi-Global Matching (SGM) method (Hirschmüller, 2008) is selected as the stereo algorithm for evaluating different matching costs because of its robustness, speed and accuracy. Four matching costs are evaluated: a parametric matching cost (AD), a non-parametric matching cost (Census), a matching cost based on Mutual Information (MI), and in addition, a new combined matching cost MI-Census (MIC). In contrast to previous studies (Hirschmüller and Scharstein, 2009; Neilso and Yang, 2008), we do not use synthetically modified datasets for performance evaluation, but use the standard Middlebury datasets as examples for close range datasets, and aerial and satellite images as examples for datasets with large baselines and stronger radiometric differences.

We focus on a fundamental question in our work: given a currently outperformed stereo method, how important is the matching cost for stereo methods on real data? We found that the performance of matching costs on the Middlebury dataset cannot be extrap- 
olated to images with larger baselines and stronger radiometric changes, and it strongly depends on the matching cost function used. A novel matching cost, linearly merged of MI and Census (MIC), shows the most robust performance during increasing length of baseline on real data.

The remainder of this paper is organized as follows: Section 2 describes the evaluated matching costs and the basics of SGM. The experiments on Middlebury datasets, airborne optical image sequences and satellite datasets are evaluated and compared in Section 3. Conclusions and the future work are presented in the last section.

\section{MATCHING COSTS AND SEMI-GLOBAL MATCHING}

\subsection{Matching Costs}

Generally, the matching costs in this work are defined on intensity, instead of color. Color channels are averaged, if they are available. Three typical matching costs are selected: a parametric matching cost (AD), a non-parametric cost (Census) and a cost based on MI. In addition, we combine linearly MI and Census with different weights to build a new matching cost (MIC).

The simplest cost function is $\mathrm{AD}$, which assumes constant intensities for corresponding pixels and, thus, lambertian reflectance and good radiometric calibration. In $1, I_{L}(p)$ and $I_{R}(p, d)$ denote the intensity of pixel $p$ in the left image and the intensity of its matched pixel at disparity $d$ in the right image separately:

$$
C_{A D}(p, d)=\left|I_{L}(p)-I_{R}(p-d)\right|
$$

In contrast to $\mathrm{AD}$, Census is a non-parametric cost. It is invariant to monotonic gray value changes and thus can tolerate a large class of global and local radiometric changes. It encodes the local image structure within a transform window and defines a bit string where each bit describes the relative ordering between the computing pixel and its local neighbor. A bit is set if a pixel inside the window has a lower intensity than the center pixel. The distance between two bit strings is computed using the Hamming distance. In our work, a $9 \times 7$ window is used and supports the matching costs in the range of 0 to 63 . $\xi$ denotes a Census transform within a window $W . \otimes$ computes the Hamming distance:

$$
C_{\text {Census }}(p, d)=\bigotimes(\underset{W}{\xi}(p), \underset{W}{\xi}(p-d))
$$

For easier combination with other costs, we rescale the matching costs into a range from 0 to 1023 .
MI combines individual entropies $H_{l}, H_{r}$ and the joint entropy $H_{l, r}$ of a stereo pair. This enables registering of images with complex radiometric relationships (Viola and Wells, 1997). In this paper, we use the Hierarchical MI (HMI) for an efficient iterative learning (Hirschmüller, 2008).

$$
M I(p, d)=H(p)+H(p-d)-H(p, p-d)
$$

The mutual information cost is also rescaled to a range from 0 to 1023 .

Due to the fixed local support, the disparity images generated using Census show slightly blurred object boundaries (Brockers, 2009). Hence, a new matching cost in this paper is a merging of MI and Census. This combination uses the advantages of both costs: the intuitive consistency and the local structure, linearly summated with different weights.

$$
\begin{array}{r}
C_{M I C}(p, d)=w_{M I} \times C_{M I}(p, d)+ \\
\left(1-w_{M I}\right) \times C_{C e n s u s}(p, d) .
\end{array}
$$

\subsection{Semi-Global Matching}

The Semi-Global Matching (SGM) method approximates a global, 2D smoothness constraint by combining many $1 \mathrm{D}$ constraints from different aggregation directions for pixelwise matching. The global energy for the disparity image is defined as $E(D)$ :

$$
\begin{array}{r}
E(D)=\sum_{P}\left(C\left(p, D_{p}\right)+\sum_{q \in N_{p}} P_{1}\left[\left|D_{p}-D_{q}\right|=1\right]\right. \\
+\sum_{q \in N_{p}} P_{2}\left[\left|D_{p}-D_{q}\right|>1\right] .
\end{array}
$$

The first term sums the costs of all pixels in the image with their particular disparities $D_{p}$. The next two terms penalize the discontinuities with penalty factors $P_{1}$ and $P_{2}$, which differ in small or large disparity difference within a neighbourhood $q$ of the pixel $p$. This minimization approximation is realized by aggregating $S(p, d)$ of path wise costs into a cost volume:

$$
S(p, d)=\sum_{r} L_{r}(p, d)
$$

$L_{r}(p, d)$ in 6 represents the cost of pixel $p$ with disparity $d$ along one direction $r$. It is described as following:

$$
\begin{aligned}
& L_{r}(p, d)=C(p, d)+\min \left(L_{r}(p-r, d),\right. \\
& L_{r}(p-r, d-1)+P_{1}, L_{r}(p-r, d+1)+P_{1}, \\
& \left.\min _{i} L_{r}(p-r, i)+P_{2}\right)-\min _{i} L_{r}(p-r, i) .
\end{aligned}
$$

This regularisation term function favores planar and sloped surfaces, but still allows larger height 
jumps in the direction of cost aggregation. The disparity at each pixel is selected as index of the minimum cost from the cost cube. In addition, $P_{2}$ is adapted to the local intensity gradient:

$$
P_{2}=\frac{P_{2} o}{1+|I(p)-I(p-1)| / W_{P_{2}}}
$$

Here, $W_{P_{2}}$ is a parameter that controls the reduction of the penalty.

\section{EVALUATION}

In this section, we test the four matching costs with SGM on Middlebury images without radiometric changes, on an aerial image sequence with increasing length of the baseline and on satellite images representing different typical object classes. In addition, we tuned the smoothness parameters of SGM for all four costs in order to get the best performance. This tuning allows concentrating on the performance of matching costs rather than the stereo method. During the evaluation, all parameters are kept constant for Middlebury images. For the airborne image sequence, an 1.7 meter resolution LIDAR 3D point cloud is used as the ground truth. A 3D point cloud acquired by the Institut Cartogràfic de Catalunya (ICC) with airborne laser scanning is used as reference data for the satellite data. The density of the point cloud is approximately 0.5 points per square meter. The data is part of the ISPRS matching benchmark (Reinartz et al., 2010).

\subsection{Results on the Middlebury Stereo Benchmark Datasets}

We apply the evaluation using on the Middlebury data. After the parameter tuning, our experimental results on the Middlebury data shown in Figure 1 are similar like the original implementations (Hirschmüller, 2008; Scharstein and Szeliski, 2011). Our post-processing steps are not tuned to the Middlebury datasets and thus our results are slightly worse results then the official SGM entry. The best parameter combinations are $P_{1}=16$ and $P_{2}=29$ for $\mathrm{AD}, P_{1}=432$ and $P_{2}=480$ for Census and $P_{1}=750$ and $P_{2}=1450$ with a $P_{2}$ adaptive factor $W_{P_{2}}=45$ for MI. Figure 1 shows the computed disparity images and the ground truth. The disparity images generated with AD have visually clean edges.

The specific error analysis is illustrated in Figure 1 bottom. The performance of MI is generally the best for all four datasets with an average percentage of bad pixels e $=9.65$ bei Error Threshold $e t=1$. Unlike
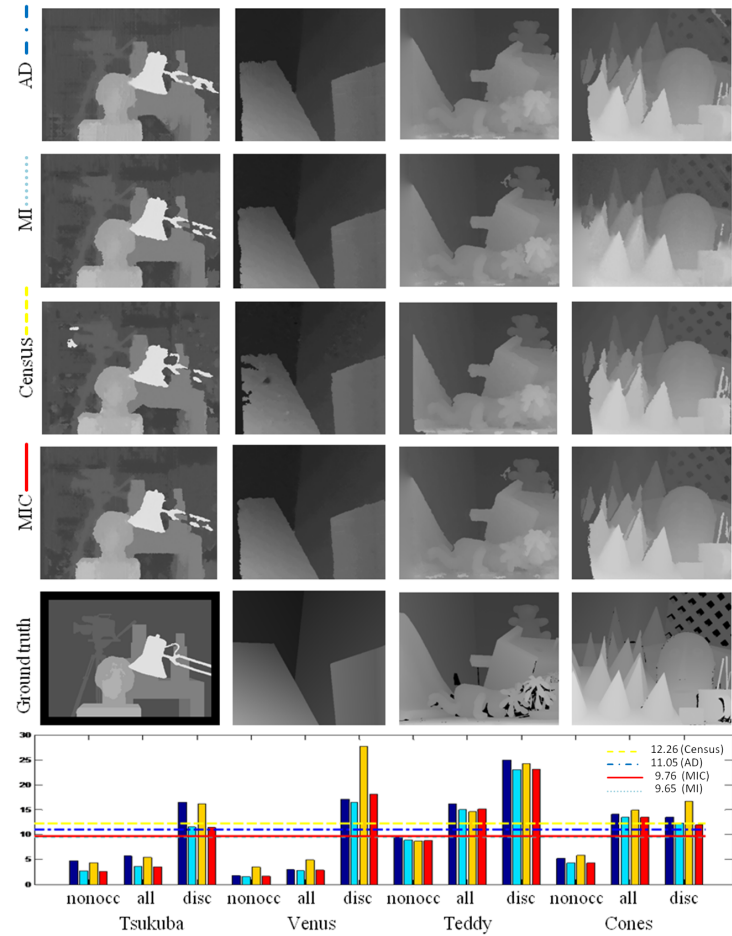

Figure 1: Results on the Middlebury Datasets for SGM with varying matching costs. Top: Depth images obtained after parameter tuning. Bottom: Bad pixel percentages for non occluded pixels, all pixels and pixels near discontinuities.

in the previous study (Hirschmüller and Scharstein, 2009), the results using Census $(e=12.67)$ are wore then using $\mathrm{AD}(\mathrm{e}=11.05)$, possibly due our basic postprocessing. We observed that the boundaries of $\mathrm{AD}$ are cleaner compared to Census, because of the fixed window size of Census. The MI reaches similar but more accurate results as $\mathrm{AD}$. On the Middlebury datasets, MIC performs similar as MI with $e=9.76$. The bast parameters for MIC are $P_{1}=750, P_{2}=1450$ and $W_{P_{2}}=50$. The cost of MI has a weight of 0.9 . This combination is better as AD, worse as MI in our study on the Middlebury data. But it outperforms the other matching costs using aerial and satellite images shown in the next subsection.

\subsection{Results on Aerial Image Sequence}

A continually recorded airborne optical image sequence is used to follow the impacts of matching costs on the performance during a changing baseline and stereo angles. The images are provided by the $3 \mathrm{~K}$ camera system, consisting of 3 Canon EOS 1D Mark II cameras with a $50 \mathrm{~mm}$ lens (Kurz et al., 2007). Only the nadir views are used in this evaluation. The flight altitude is approximately 1500 meters above 
ground. The distance between each recorded observation is about 35 meters. The largest baseline we present in this work is about 250 meters.

Figure 5 visualizes the impact of larger baselines on the matching performance clearly: although the results computed on images with small baselines perform well using all four matching costs, the errors of $\mathrm{AD}$ rise quickly with increasing baseline and stereo angle, possibly due to the non-lambertian reflectance. Hierarchical MI (HMI) does perform slightly better than $\mathrm{AD}$ for small baselines, and the disparities in the street are smoother and show less noise, c.f. Figure 3. For small baselines, Census does produce a noise disparity map, but shows the most robust behaviour for larger baselines, as visible on the church roof in Figure 5.

In addition, we observed that the results using MI have sharper edges at discontinuities. In contrast, the fixed local support of Census causes slightly blurred edges. In Figure 2, the comparison on details between disparity images using different matching costs are shown. The smoothness on surfaces of the results using $\mathrm{MI}$ is generally better compared to $\mathrm{AD}$ and Census. A visual analysis is illustrated in Figure 3.

Because of the advantages of MI for sharp edges and smoothness on surfaces, and the robustness of Census, we combine MI with Census to generate matching the cost MIC. It performs similar to MI at object discontinuities, but keeps the robustness of Census for larger baselines.

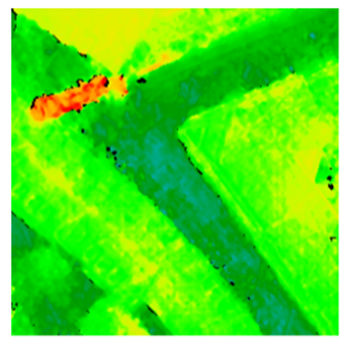

$\mathrm{AD}$

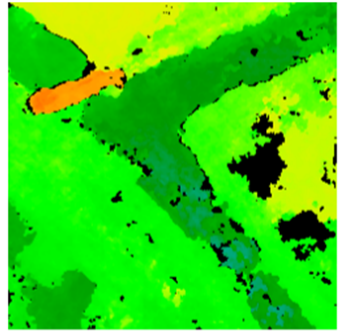

Census

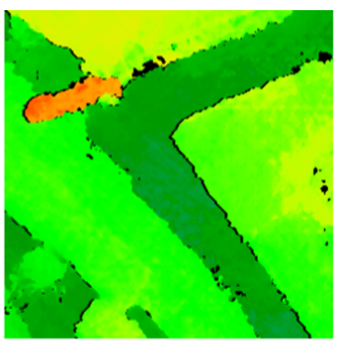

MI

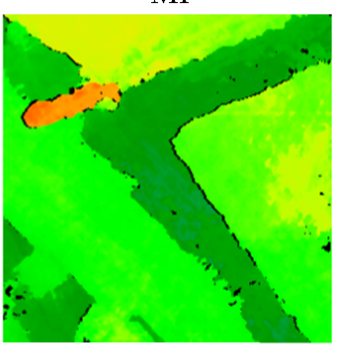

MIC
Figure 2: Comparison on details of disparity images using different matching costs: MI and MIC show better object boundaries and less noise. Different colour codifications denote different disparities of corresponded pixels.
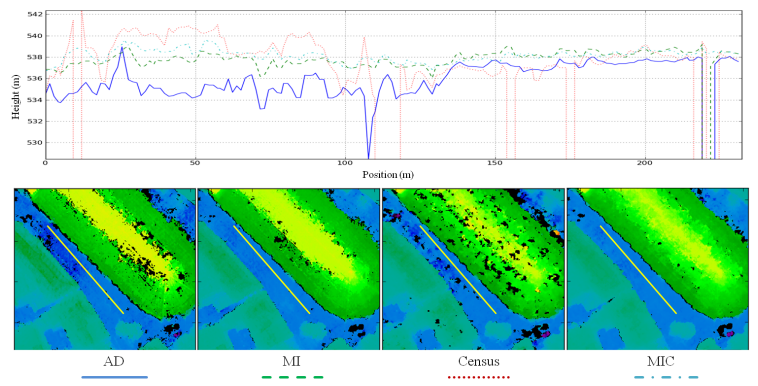

Figure 3: Comparison of smoothness for AD, MI, Census and MIC results on a flat street. The diagram shows the height values along the profile indicated by the yellow line.

\subsection{Results on Satellite Data}

We evaluated the matching costs on a Worldview-1 stereo image pair with a ground sampling distance of $50 \mathrm{~cm}$, and a relatively large stereo angle of $35^{\circ}$. The data is part of the ISPRS matching benchmark (Reinartz et al., 2010). A small cutout of the stereo data and the reconstruction results for an urban area (Terrassa in Spain, Barcelona) are shown in Fig. 4. The full dataset covers mountainous, agricultural, forest, industrial and residential areas. The figure indicates that these images cannot be matched successfully using MI, while Census and the MIC perform reasonably well on this challenging dataset. The large black background in the MI image was incorrectly filled using this data. Table 1 shows the results of evaluating the city area shown in Fig. 4 and two other test areas (hilly forest and industrial area) against the LIDAR reference data. It is clearly visible that MIC performs slightly better than Census and that MI does produce the largest errors. Experiments with various values for $P_{1}, P_{2}$ and $W_{P_{2}}$ indicated that performance depends mostly on the cost function and not on the exact parametrisation of the stereo algorithm.

\begin{tabular}{l|cccccc} 
Cost & $P_{1}$ & $P_{2}$ & $W_{P_{2}}$ & $w_{M I}$ & NMAD & BP $(\%)$ \\
\hline & & & & & & \\
MIC & 700 & 1400 & 200 & 0.3 & 0.72 & 15.8 \\
Census & 600 & 1300 & 200 & - & 0.74 & 16.8 \\
MI & 700 & 1400 & 200 & - & 1.10 & 25.8
\end{tabular}

Table 1: Evaluation of Matching results in three test areas against ground truth LIDAR Data. NMAD is the normalized median deviation and BP (Bad Pixels) is the percentage of pixels with an absolute height error $>2 \mathrm{~m}$. 


\section{CONCLUSION AND FUTURE WORK}

In this work, three typical matching costs (AD, MI and Census) and a novel matching cost (MIC) are evaluated using SGM on the Middlebury stereo benchmark, aerial and satellite images with ground truth. In summary we found that the performance of dense stereo methods for datasets with larger baselines and stronger radiometric changes strongly relies on robust matching costs. The experimental results on different data sets show, AD works well for scenes with a small baseline and a good radiometric calibration. MI performs slightly better than Census in case of low radiometric changes and stereo pairs with small baselines, but fails in areas with local radiometric changes. MI keeps sharp edges at discontinuities and results in less noisy disparity maps when compared to Census. Census performs well for larger baselines, but results in slightly blurred object boundaries. For large baseline satellite stereo imagery Census performs significantly better than MI. The weighted sum of MI and Census (MIC) unifies the advantages of MI and Census and outperforms $\mathrm{AD}, \mathrm{MI}$ and Census on remote sensing datasets.

Topics for future work include developing a methodology for evaluation of remotely sensed images against LIDAR ground truth. The main challenges for this task are changes due to multi-temporal data acquisition and different resolution and behaviour of the sensors. The influence of different stereo algorithms on the performance of the matching cost functions will be evaluated in further work.

\section{ACKNOWLEDGEMENTS}

Special thanks are given to the data providers for the provision of the stereo data namely: Digital Globe for the Worldview-1 data and ICC Catalunya for the reference data.

\section{REFERENCES}

Birchfield, S. and Tomasi, C. (1998). A pixel dissimilarity measure that is insensitive to image sampling. IEEE Transactions on Pattern Analysis and Machine Intelligence, 20(4):401-406.

Brockers, R. (2009). Cooperative stereo matching with color-based adaptive local support. Computer Analysis of Images and Patterns.

Chrastek, R. and Jan, J. (1997). Mutual information as a matching criterion for stereo pairs of images. Analysis of Biomedical Signals and Images, 14:101-103.
Hirschmüller, H. (2008). Stereo processing by semi-global matching and mutual information. IEEE Transactions on Pattern Analysis and Machine Intelligence, 30(2):328-341.

Hirschmüller, H. and Scharstein, D. (2009). Evaluation of stereo matching costs on image with radiometric differences. IEEE Transactions on Pattern Analysis and Machine Intelligence, 31(9):1582-1599.

Kurz, F., Müller, R., Stephani, M., Reinartz, P., and Schroeder, M. (2007). Calibration of a wide-angle digital camera system for near real time scenarios. ISPRS Workshop High Resolution Earth Imaging for Geospatial Information.

Neilso, D. and Yang, Y. (2008). Evaluation of constructable match cost measures for stereo correspondence using cluster ranking. IEEE Conference on Computer Vision and Pattern Recognition.

Reinartz, P., d'Angelo, P., Krauß, T., Poli, D., Jacobsen, K., and Buyuksalih, G. (2010). Benchmarking and quality analysis of dem generated from high and very high resolution optical stereo satellite data. ISPRS Symposium Commission I.

Scharstein, D. and Szeliski, R. (2002). A taxonomy and evaluation of dense two-frame stereo correspondence algorithms. International Journal of Computer Vision, 47(1):7-42.

Scharstein, D. and Szeliski, R. (2011). Middlebury stereo vision research page. http://vision.middlebury . edu/stereo/.

Viola, P. and Wells, W. M. (1997). Alignment by maximization of mutual information. International Journal of Computer Vision, 24(2):137-154.

Zabih, R. and Woodfill, J. (1994). Non-parametric local transforms for computing visual correspondancen. In Proc. European Conference of Computer Vision. 


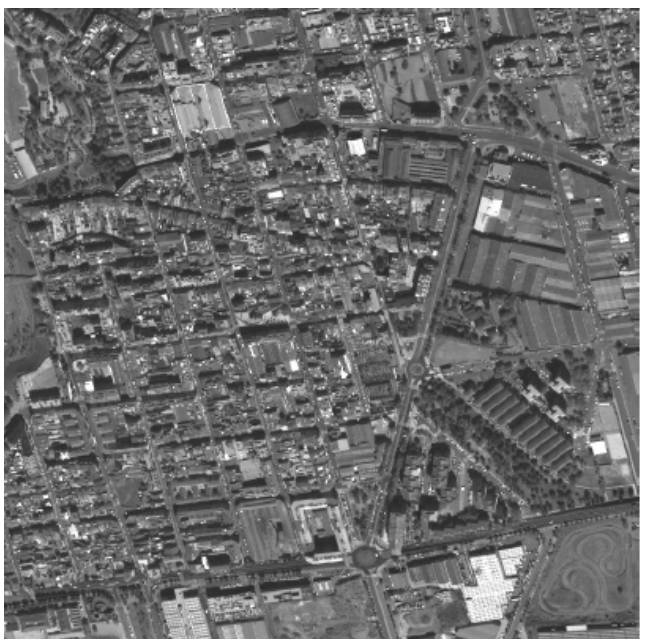

Image 1

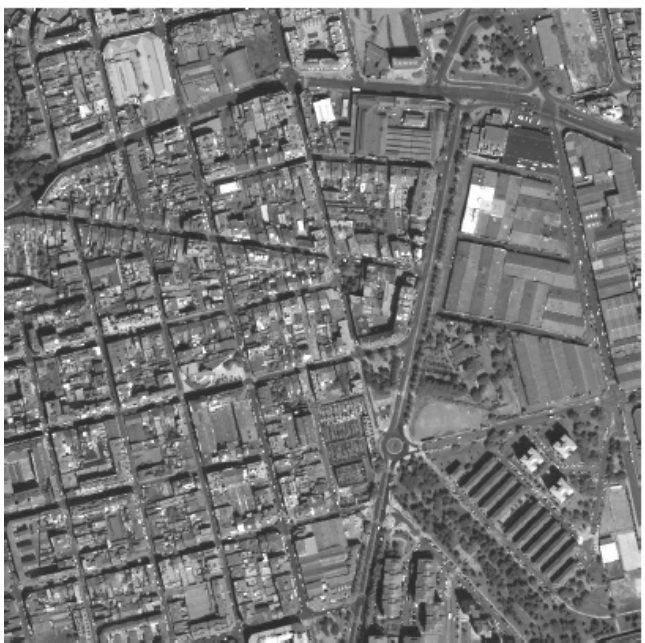

Image 2

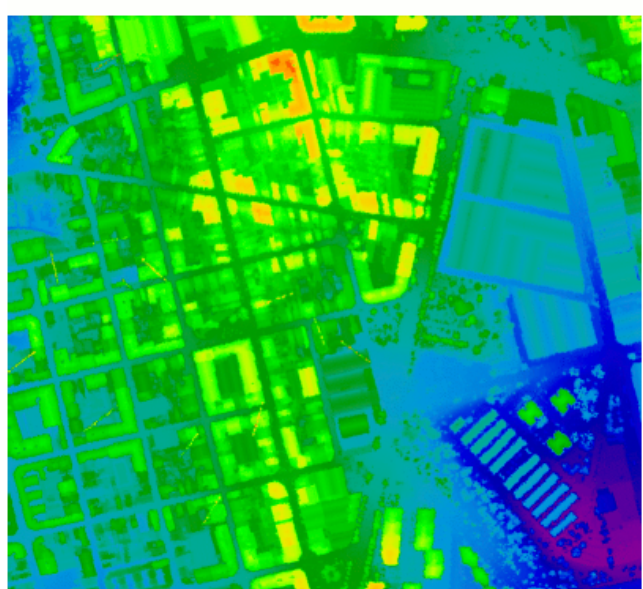

LIDAR reference

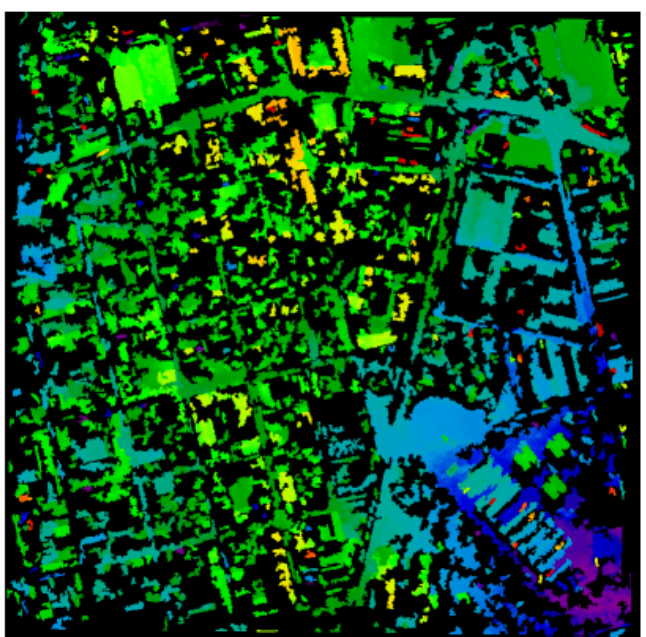

MI

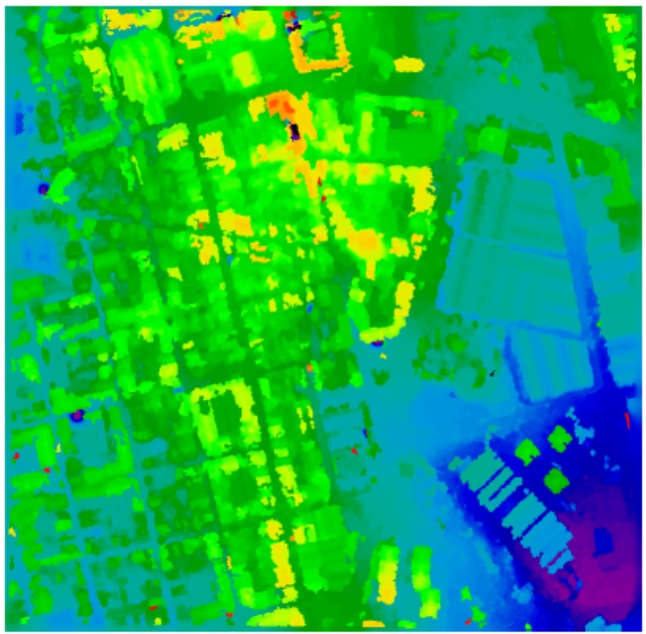

Census

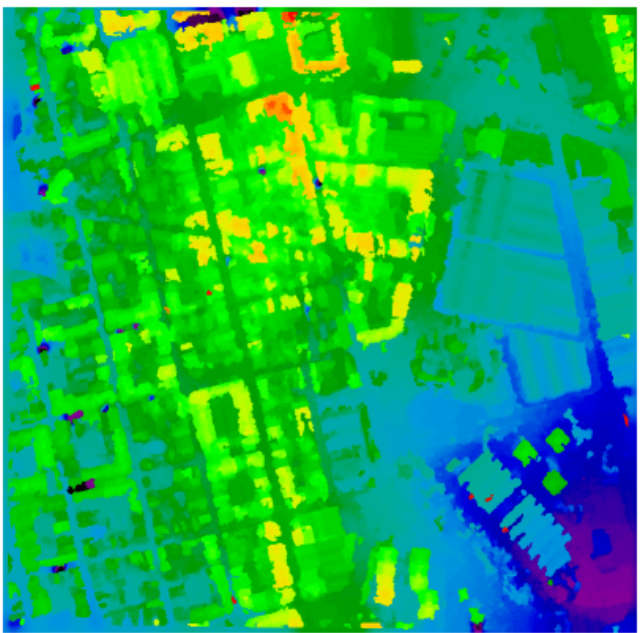

MIC

Figure 4: Small cutout of the Worldview-1 Stereo pair. First row: stereo pair and LIDAR reference data. Second row: Results after stereo matching with different cost functions, orthographic reprojection and discontinuity preserving interpolation. 

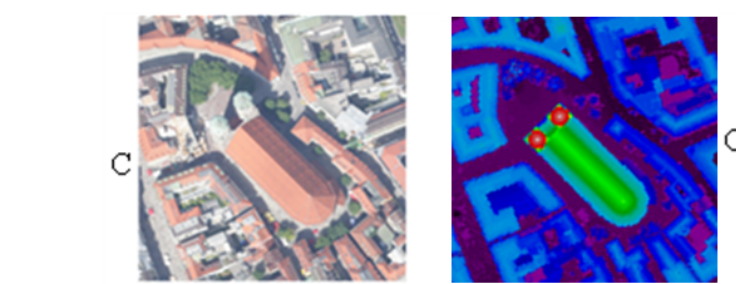

Ground truth
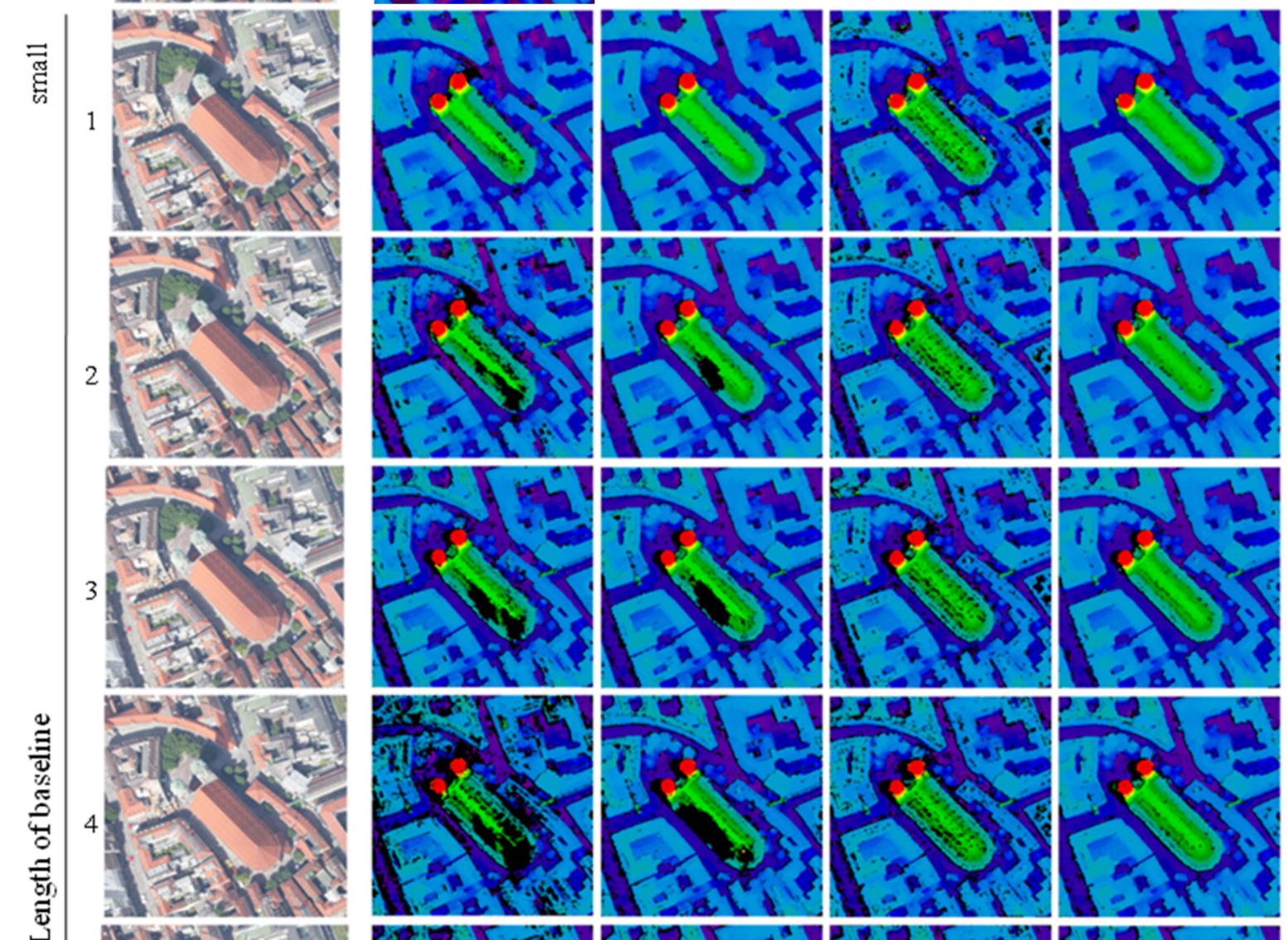

ब
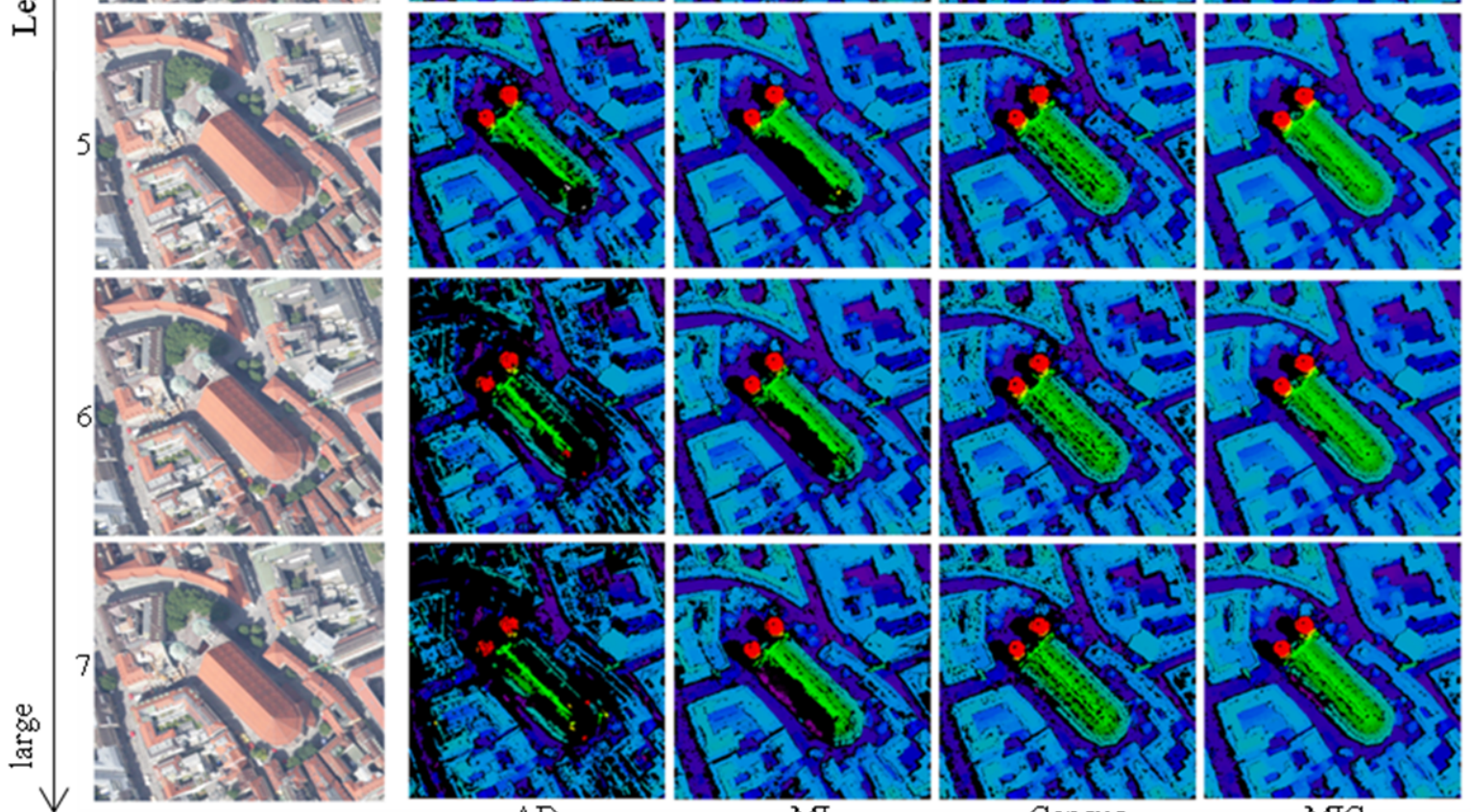

Figure 5: Disparity maps for stereo pairs with increasing baseline. The images 1 to 7 are matched with the centre image $C$. The results for AD, MI, Census and MIC are shown in columns 2-5. The black areas indicate failures of the left-right check. The ground truth DSM (Digital surface model) from laser scanning is shown next to the centre image 